\title{
LEBESGUE ON INTEGRATION
}

Leçons sur l'Intégration et la Recherche des Fonctions Primitives. By H. Lebesgue. Second edition. Paris, Gauthier-Villars, 1928. xv+342 pp.

A second edition of Lebesgue's book on integration after almost twentyfive years! Is it going to be a treatise on Lebesgue integration, following the lead of some recent books on this subject, or will it be in fact a second edition, i.e. a continuation and bringing up to date of the theme which dominated the first edition? The preface already gives enlightenment on this question. We find that Lebesgue has not yielded to the temptation of going into the details of the Lebesgue type of integration and its application, but is more interested in giving a logical continuation of the first edition of the book. He apologizes for omitting so many things which might be of interest, simply because they do not belong. The first edition is almost entirely contained in this second one, even to the extent of misprints, but the book has grown to almost three times its original proportions.

The new edition still forms one logical whole. The underlying problem and theme of the book is still to be found on the first page: "To find the functions $F(x)$ which admit as a derivative a given function $f(x)$." It is this theme with its variations, extensions and elaborations which forms the subject matter of the book. The author, in true composer fashion, begins with a simple statement of the theme, produces the harmony contained in the researches of Cauchy, which gives the wonderfully esthetic result that every continuous function is the derived function of another continuous function, carries it through the extension to Riemannian and related integrations, the introduction of Lebesgue integration, and as an added and extended feature, treating not only the derived functions but the upper and lower derivates. More and more complex become the variations as we approach the contribution made by Denjoy in his totalization, from which Lebesgue extracts in a triumphant fashion the important theorem on the possible distributions of the values of the derivates of a continuous function. Once again the theme starts simply and casually with a new kind of harmonic structure, the Stieltjes Integral puts in its appearance and through a series of complexities, one is led to a grand finale of the notion of derivatives with respect to functions of bounded variation and a totalization process as applied to these. As one applies a critical analysis to this composition as a whole, one cannot help but admire the skill and artfulness with which the entire structure is built, how each item contributes its share to the whole, how each consideration leads on to further results, and plays its own part in the sequel. It is indeed wonderful how the material drawn from the first edition seems in some farsighted way to have been a fitting preliminary to the later developments and takes its place as an integral and inseparable part of the whole.

The first six chapters of the book are to a large extent identical with the corresponding chapters in the first edition, additions being mainly in the footnotes, and to some extent in the text, where the later chapters seemed to require more extended treatment of topics, and where the intervening years have 
brought new worthwhile results. These chapters take one through the Cauchy definition of integration, that is, the integration of continuous functions, the Riemann definition, the geometric definition of integration, functions of bounded variation - this topic extended slightly to include a more extended study of the convergence of the variational sums to the total variation-and a study of the derived numbers of continuous functions and questions of the determination of a function by its derivates. ${ }^{*}$

These chapters have been discussed in the review of the first edition of the book (this Bulletin, vol. 14 (1908), pp. 501-4). We subscribe to the opinions there expressed, except that in the intervening years the material of the first edition has become a natural part of our mathematical thinking and seems simpler than when first propounded. In going over this section we were rather impressed by the variety of results concerning derivatives and derivates obtainable without the use of integration, e.g., the theorems which give the leeway in the determination of a function by its derivates, the theorem that a uniformly convergent sequence of derivative functions is again the derivative of a continuous function, and so on. Although Lebesgue later on makes a point of obtaining results on derivatives by studying the inverse process, he gives in these early chapters a fine illustration of how much can be accomplished by direct methods.

The seventh chapter gives an exposition of the Lebesgue method of integration, and follows the manner of treatment in the first edition, that is, starting with the given properties of integration, he derives a form which these integrals must take. The main additions in this chapter are more explicit proofs of the fact that the integral obtained by using the properties specified actually does have the desired properties, an addition which is highly desirable. Of the many other methods of defining the Lebesgue type of integration, he singles out one of the early definitions of W. H. Young, which follows the Riemannian type of definition, subdivisions being into measurable sets instead of intervals. It is perhaps interesting to remark that Lebesgue's definition lends itself most readily to proofs of properties relating to additivity of the integral as a function of sets, while the Young type lends itself to the additivity as a function of functions.

The eighth chapter, entitled The indefinite integral of summable functions, is devoted to an exposition of completely additive functions of measurable sets, suggested by the fact that the integral is such a function of sets. The author distinguishes between three types of indefinite integral, $\int_{a}^{x} f(x) d x+$ const.; $\int_{\alpha}^{\beta} f(x) d x,(\alpha, \beta)$ being any subinterval of $(a, b)$; and $\int_{E} f(x) d x$ where $E$ is any measurable subset of $(a, b)$. He amuses himself by playing with the words indefinite and definite, e.g., "It is clear that in the expressions definite and

* It seems unfortunate that some of the misprints and errors of the first edition were not corrected in the second. The reviewer is puzzled as to why (p. 78) the function $x^{2} \sin (1 / x)$ should be cited as an example of a function of bounded variation with unbounded derivative, why (p. 102) the continuous function $x\left(x^{2} \sin (1 / x)\right)^{\prime}$ is not integrable $D$, i.e. the derivative of a continuous function, and why (p. 139) $m_{l, i}(\phi \geqq \alpha)$ is con inuous on the left in $\alpha$ since it is not in general true that if the sets $E_{n}$ form a monontonic increasing sequence converging to $E$, then the lower measure of $E_{n}$ approaches that of $E$. 
indefinite integral, indefinite is not in the sense of infinite, but of not defined. Definite has the sense determined. $\int_{\alpha}^{\beta} f(x) d x$ is definite if the interval of integration is defined, but indefinite when $(\alpha, \beta)$ is undefined, not definite, not determined, unknown, variable." And later in a footnote (p. 195), in which he takes exception to Denjoy's introduction of new words like "épaisseur" as opposed to "density at a point,"* he concludes with "however that may be the reader who has followed me up to this point will not be troubled by the question of words, since he has consented to engage in investigating the definition of the definite integral, which might seem ridiculous, and the definition of the indefinite integral, which remains indefinite even after it has been defined, which is a little humiliating."

The main portion of this chapter is devoted to the separation of an absolutely additive function of sets into three parts, the function of the discontinuities, the absolutely or completely continuous portion, and the function of singularities, together with the properties of the sets of singularities, the treatment suggested to a large extent by the work of de la Vallée Poussin. What worries one particularly in this chapter is that it is not stated explicitly at the beginning of the investigation to what class of sets these additive functions apply, and it is only by reading between the lines that one concludes that the author is interested in additive functions of measurable sets.

Chapter IX is concerned mainly with the derivative properties of the indefinite Lebesgue integral. By setting up the integration process for a derivate of a continuous function he obtains the result that a continuous function of bounded variation has a finite derivative except at a set of measure zero, and derives the relation between the variation of the function on an interval and the integral of its derivative, yielding the well known necessary and sufficient conditions that a function be an indefinite Lebesgue integral as well as the relationship between a function and its integrable derivatives. The method of procedure, while perhaps consistent and logical in its setting is rather involved, playing tag with $\epsilon^{\prime}$ 's approaching zero, and $M$ 's approaching infinity, to an extent to make it seem almost uncertain that the theorems have actually been proved. From the point of view of elegance the method of de la Vallee Poussin, involving the majoring and minoring functions, is far superior. The later parts of this chapter extend the theorem on the existence of a derivative of a function of bounded variation to the discontinuous case, the method being via results on integrals. There then follows a brief exposition of derivatives at a point as defined by the quotient of a set function by the measure of a set. There is a delightful explanation of how the desire to have

* When it comes to a question of terminology, the reviewer has his doubts as to the advisability of introducing a new terminology for each new method of defining integration. The use of the word summable, transliterating the French "sommable," for Lebesgue integration, interferes with the word usually applied to a process relating to infinite series. Is Lebesgue integration a summability process applied to functions not Riemann integrable? And then the word totalization for Denjoy's definition. Why total? I prefer to use the word integration for all such processes and, if distinctions are needed, to characterize them by special names, Riemann, Lebesgue, Denjoy, Stieltjes. 
the values of the function near a point be the main contributors in the value of the derivative leads inevitably to the notion of order of a set at a point. The chapter closes with a brief treatment of length of arc, a subject which assumes a place in the background in this edition.

The tenth chapter is devoted to a presentation of the subject of totalization, i.e., the Denjoy extension of the Lebesgue integration. The presentation of the material is such that one gains an insight into this process which the writings of Denjoy and others have not succeeded in conveying. Perhaps the easy steps through which the ultimate exposition of the totalization process in all its generality is reached contribute no small share. In order to get the reader familiar to some extent with the transfinite operational process involved, we have as the first section of this chapter a proof of the Baire Theorem concerning the nature of functions of the first class. This theorem illustrates two points which are made fundamental to the later considerations, (a) that a well ordered set of closed sets each of which is non dense on the preceding terminates after a denumerable number of steps, (b) if for a given operative process $O$, it is known that for every closed set $E$ belonging to $(a, b)$ there exists a subinterval $(l, m)$ containing points of $E$ on which the operation $O$ may be defined, then the operation $O$ can be defined on $(a, b)$.

In discussing totalization the first type of function considered is assumed to be the finite valued derivative $f(x)$ of a continuous function $F(x)$. For such a function $f(x)$ it is proved that for every closed set $E$ there exists an interval $(l, m)$ containing points of $E$, so that $f(x)$ is bounded on the part of $E$ in $(l, m)$ and the sum $\sum[F(\beta)-F(\alpha)]$ extended over the intervals $(\alpha, \beta)$ complementary to $E$ relative to $(l, m)$ is absolutely convergent, making it possible to define $F$ on the interval $(l, m)$ if it is known on the intervals complementary to $E$, by the formula $\int_{E} f(x) d x+\sum[F(\beta)-F(\alpha)]$. Then come finite valued functions $f$, which are known to be one of the derivates (right hand, upper or lower, or left hand, upper or lower) of a continuous function, and in that case the boundedness of $f$ on the part of $E$ in $(l, m)$ is replaced by the summability. The process of totalization then divides itself into two parts, one part to enable one to extend it step by step over a set of intervals each containing the preceding, the other involved in the formula quoted above. There follow theorems giving conditions under which a function is totalizable, and also some giving necessary and sufficient conditions in order that a continuous function $F(x)$ be an indefinite total, the most elegant of these latter theorems being that for every closed set $E$ the function $G(x)$ equal to $F(x)$ on $E$ and linear on the complement of $E$ is absolutely continuous in some interval containing points of $E$. It is to be noted that the totalization process which is treated here is not the one originally proposed by Denjoy. This is a weaker definition and therefore more generally operative. The first totalization process demanded certain absolute convergences, including the convergence of the oscillations (not variations) of the function on the complementary intervals of closed sets. This first type of totalization has properties much more closely allied with those of the simpler integration. In order to get derivative properties for the general totalization it is necessary to introduce the notion of approximative derivative at a point, i.e. the derivative of a function $F(x)$ on a set of density 1 at the point. The final section of the chapter gives the elegant theorem of Denjoy on the 
possible distribution of the values of the four derivates of a continuous function, together with the interesting result that if one knows that a finite valued function $f(x)$ is at each point one of the four derivates of a function $F(x)$, not always the same derivate, then the function $F(x)$ is determined from $f(x)$ by the totalization process. Totalization, then, as the indirect process can accomplish for the derivatives of a continuous function results derivable also by studying these derivates directly. Obviously it does not extend to discontinuous functions, where direct methods are more powerful and effective. Totalization does accomplish about all that one has a right to expect for the inversion of finite valued derivates, the path for the inversion of the infinite valued derivate being obviously blocked by the inability to distinguish between infinites.

The chapter on Stieltjes integrals begins with a discussion of what might be called the Stieltjes integral, where, in $\int f d \alpha, f$ is continuous and $\alpha$ of bounded variation. Thus $\int f d x, f$ continuous, might be called the Cauchy integral. In listing the properties of these integrals Lebesgue strangely enough omits explicit mention of the linearity in $\alpha$ which is used perhaps more than any of the others Much time and effort is devoted to reducing the Stieltjes integral to a Lebesgue integral by transformation, but Lebesgue fails to comment on the fact that his own definition of integral is really a Stieltjes integral, $\int_{m}^{M} y d \mu(y)$ where $\mu(y)$ is the measure of the set $E(f>y)$, and $(m, M)$ is an interval enclosing the interval of variation of $f$. It seems to me however that in such transformations one loses sight of important properties. It certainly is not a very easy matter to deduce properties of Lebesgue integration from its expression as a Stieltjes integral, and the properties of the function $\mu(y)$ associated with a given function. The marvel is how many of the properties and extensions of Stieltjes' integrals can be derived from their Lebesgue integral transform.

There is a lack of clearness in the treatment of the so-called RiemannStieltjes integral; why a new terminology is necessary is not obvious. To the reviewer the proof of the theorem that a necessary and sufficient condition that a function $f$ be Stieltjes integrable with respect to a function of bounded variation $\alpha$ is that the set of points of discontinuity of $f$ have a total variation zero with respect to $\alpha$, is not at all convincing. There seems to me to be a difficulty regarding the limits processes involved. Essentially there are, in integration definitions of the Riemann type, two methods of approach, one as to a norm, viz. the maximum of the length of the intervals in a subdivision, the limit being in the sense that the norm approaches zero, the other a limit as to subdivisions, monotoneity of approach being obtained by assuming that each subdivision is a redivision of the preceding.* The Riemann integration is an instance of the first type, the Darboux integrals exist after the second manner, and the conditions of integrability via the upper and lower integrals are dependent upon the proof of the fact that the Darboux integrals are also existent as limits as to a norm. The total variation of a function exists as a limit after the second manner, and after the first only if the function has only regular points, that is, for every $x, \alpha(x)$ lies between $\alpha(x+0)$ and $\alpha(x-0)$. The upper and lower in

* See for instance, E. H. Moore, Proceedings of the National Academy, vol. 1 (1915), pp. 628-32, American Journal of Mathematics, vol. 44 (1922), pp. 102-114. 
tegrals in a Stieltjes integral exist after the second manner, but in this case there is in general no theorem corresponding to the Darboux theorem unless $\alpha$ is continuous. Lebesgue modifies the norm definition by requiring that the points of discontinuity of $\alpha$ be gradually made to be the points of division, so as to secure an equivalent of the second mode of definition, which method of approach is obviously limited to the case in which the points of discontinuity of the function $\alpha$ are denumerable.

After a brief reference to the notion of Stieltjes integrals in the applications, there follows the definition of the derivative of a function with respect to a function of bounded variation, boldly set down as the limit of $\Delta F / \Delta \alpha$ as $x$ approaches $x_{0}$, without any particular worry about the behavior of the function $\alpha$ except for its bounded variation character. This leads up to the notion of a totalization process extended to a Stieltjes integral. Lebesgue seems loath to abandon the condition that the function $\alpha$ be of bounded variation, because "one cannot attach to every continuous function an integral with respect to $\alpha$ if $\alpha$ is not of bounded variation." But just why continuous functions should be singled out in this way is not clear. This section is to some extent rather sketchy but on the other hand very suggestive, and will undoubtedly give rise to interesting further developments. It forms a fitting close to the main part of the book.

The book closes with an appendix of about twenty-five closely printed pages, on the subject of transfinite numbers. It gives an interesting introduction to this topic, interspersed with comments and cogitations on the subject and its applicability. There are many worthwhile things. Perhaps the nature of some of the cogitations will be evident from some quotations. In connection with the reasoning by transfinite induction he says: "Must one then accept this method of demonstration? Not at all, it is up to each one to decide for himself whether or not he is satisfied with reasoning by transfinite induction. Further as almost everything in mathematics has been written only for those who admit the reasoning by ordinary induction so certain passages of this book are written only for those who permit reasoning by transfinite induction." In discussing the matter of replacing proofs by the use of transfinite induction by proofs avoiding this method, he distinguishes sharply between the theorem of Cantor-Bendixson, that every closed set is the sum of a perfect set and a denumerable set, and the problem of Cantor-Bendixson to decompose a closed set into the sum of a perfect set and a denumerable set; similarly the theorem of Baire, that every function of the first class is pointwise discontinuous on every perfect set, and the problem of Baire, for a function pointwise discontinuous on every perfect set, to set up a sequence of continuous functions of which it is the limit. He continues: "In the researches of Denjoy, the very definition of the operation of totalization requires the transfinite, and one cannot hope to avoid its use. But nothing prevents one from hoping that it is possible to replace the operative process of Cantor-Bendixson, of Baire, and of Denjoy by nontransfinite processes permitting one to solve the problems of Cantor-Bendixson, Baire, and 'primitive' functions respectively. In fact it is possible to demonstrate the theorem of Cantor-Bendixson, and of Baire, and to solve the problem of Cantor-Bendixson, without the use of the transfinite induction."

\section{T. H. HildeBrandT}

